\title{
A Survey on Rural Broadband Wireless Access Using Cognitive Radio Technology: IEEE 802.22 Wireless Regional Area Networks
}

\author{
M.Ravi Kumar*, S.Manoj Kumar, M.balajee \\ Dept. of IT (MCA), G M R Institute of Technology Rajam, Andhra Pradesh, India \\ *Corresponding Author: ravi.mrk91@gmail.com
}

Copyright (C) 2013 Horizon Research Publishing All rights reserved.

\begin{abstract}
The previous and most popular broadband wireless technology i.e. WiMAX which is limited about to 10 miles, there are power and line of sight issues yet to be resolved for a broader coverage area. WiMAX deployment is therefore limited to densely populate metropolitan areas. What about rural and sparsely populated, geographically dispersed regional areas? Here is the upcoming solution for that which is implemented by IEEE .The IEEE 802.22 standard defines a system for a Wireless Regional Area Network i.e. WRAN that uses unused or white spaces within the television bands between 54 and $862 \mathrm{MHz}$, especially within rural areas .The 802.22 standard is the first standard to adopt cognitive radio spectrum sensing as a means of gaining greater use of the radio spectrum. Cognitive radios are to provide broadband wireless access (BWA) in the licensed TV bands on a secondary access basis. This concept is examined to see under what conditions BWA could be viable. Rural areas require long range communication which cognitive radios may not be able to support with enough secondary spectrum. By using cognitive radio networking techniques, it is able to sense the environment and adjust the network to accommodate any changes. In this paper we present the complete overview and broad survey of this CR based WRAN technology, how it will be fulfill the upcoming challenges and other factors and some survey based comparisons between and IEEE 802.16 WiMAX and IEEE 802.22 WRAN. Also we list some of the minor and major security issues regarding to this IEEE 802.22 standard technology.
\end{abstract}

Keywords WRAN, , Self-Co-existence Cognitive Radios, Sensing, WiMAX, Hidden Incumbent Problem

\section{Introduction}

A Wireless Regional Area Network (WRAN) system based on 802.22 protocols is intended to make use, on a non-interfering basis, of unused TV broadcast channels
$(6 \mathrm{MHz}, 7 \mathrm{MHz}$, or $8 \mathrm{MHz})$ to address, as a primary objective, rural and remote areas and low population density underserved markets with performance levels similar to those of Broadband access technologies such as digital subscriber line (xDSL) technologies and Digital Cable modem service. A secondary objective is to have this system scale to serve denser population areas where spectrum is available. The WRAN system must be capable of supporting a mix of data, voice and audio/video applications. These include Internet access, VoIP, video teleconferencing and streaming video. In the USA, not all the TV channels are used as it is necessary to allow guard bands between active high power transmitters to prevent mutual interference. Also not all stations are active all of the time. Therefore by organizing other services around these constraints it is possible to gain greater spectrum utilization without causing interference to other users [1]. The remaining explanations could be given in the following sub sequent sections.

The idea behind 802.22 is that there are considerable unused frequencies between VHF and UHF broadcast channels between 54 and $862 \mathrm{MHz}$ this is possible by using cognitive radio capabilities. These include dynamic spectrum access, incumbent database access, accurate geo location techniques, spectrum sensing, spectrum etiquette, and coexistence for optimal use of the available spectrum. Essentially this means that it will be possible to send wireless broadband access without interfering with TV signals. It is designed for last-mile service in low populated areas especially rural area. The CR base station covers an area between $33 \mathrm{~km}$ (typical) to $100 \mathrm{~km}$ and the network is designated to provide the minimum throughput of $1.5 \mathrm{Mbps}$ for the downstream and $384 \mathrm{kbps}$ for the upstream. In additional to conventional PHY and MAC layer functionalities, new features have been included in IEEE 802.22standard.

The WiMAX (IEEE Standard 802.16e) technology is widely used now-a-days whereas WRAN (IEEE Standard 808.22) is a developing project of IEEE which was finally published in July 2011. 


\section{Existing Technologies}

There are numerous documented research papers on possible ways of providing internet connectivity to rural areas using existing technologies. These technologies deployed include the use of: Satellite, Cellular, Wi-Fi, WIMAX and Hybrid Broadband Access networks to name just a few. These technologies have their pros and cons but the major drawback for all of them hinges on the affordability of the end user to take up the service; for the rural market these networks would be unviable hence unsuitable for rural areas. However new ways continue to be sought in order to bridge the financial gap using combinations of these existing technologies e.g. use of Wireless Mesh Networks.

\section{Related Work}

The IEEE 802.22 activity is the first worldwide effort to define a standardized air interface based on CR techniques for the opportunistic use of TV bands on non-interfering basis. Due to this fact, the work being done in IEEE 802.22 is in many respects unique and the first of its kind, with little relevant related work. Its development process is a combined effort of traditional companies as well as representatives from the incumbent community (TV broadcasting and Wireless Microphones), and is scheduled to produce version 1.0 of the draft standard around January 2007. It is important to understand, however, the core differences between 802.22 and 802.16 (WiMAX) as confusion often arises when discussing these two IEEE projects. 802.22 is mostly targeted at rural and remote areas and its coverage range is considerably larger than 802.16 Also, 802.16 does not include incumbent protection techniques necessary to operate in licensed bands, while it has an ongoing project (802.16h) currently concentrating on coexistence among 802.16 systems only [6].

\section{Background}

The IEEE 802.22 standard for a Wireless Regional Area Network or WRAN system has been borne out of a number of requirements, and also as a result of a development in many areas of technology. In recent years there has been a significant proliferation in the number of wireless applications that have been deployed, and along with the more traditional services this has placed a significant amount of pressure on sharing the available spectrum. Coupled to this there is always a delay in re-allocating any spectrum that may come available.

One particular technology that is key to the spectrum deployment of new services that may bring better utilization is that of cognitive radios technology. By using this radios can sense their environment and adapt accordingly. The use of cognitive radio technology is therefore key to the new IEEE 802.22 WRAN standard [2].
WiMAX (IEEE Standard 802.16e) overview [6]: WiMAX (Worldwide Interoperability for Microwave Access) is a standards-based wireless technology that provides high-throughput broadband connections over long distances and mobile environment. The IEEE $802.16 \mathrm{e}$ is based on Orthogonal Frequency Division Multiple Access (OFDMA) whose main aim is to give better performance in non-line-of-sight (NLOS) environments. IEEE 802.16e introduced scalable channel bandwidth up to $20 \mathrm{MHz}$, Multiple Input Multiple Output (MIMO) and MAC enabled $802.16 \mathrm{e}$ technology to support peak downlink (DL) data rates up to $63 \mathrm{Mbps}$ in a $20 \mathrm{MHz}$ channel through Scalable OFDMA (S-OFDMA) system.

\subsection{Introduction to Wireless Standard Based on Cognitive Radios}

The industry definition of a cognitive radio is a device that, unlike a traditional radio, can dynamically find and use available frequency to improve throughput and connectivity. This can be done via real-time sensing that allows the radio to scan for unused frequencies and then instantly tune to them. Cognitive radios can also rely on a database that can tell it what channels are available (usually based on the radio's location and known spectrum restrictions in that area). Either or both of these techniques can be used to help the cognitive radio avoid interference and optimize its throughput and connection reliability on a dynamic basis. With detailed information about its local RF environment, smart radios are able to change power output, frequency and receive or transmit parameters, in order to extract latent (unused) bandwidth and capacity from crowded unlicensed, as well as underutilized licensed wireless spectrum.

Cognitive Radios (CRs) are seen as the solution to the current low usage of the radio spectrum. It is the key technology that will enable flexible, efficient and reliable spectrum use by adapting the radio's operating characteristics to the real-time conditions of the environment. IEEE 802.22 standard is preferred for two main reasons: (a) no dedicated spectrum is required, hence service providers can save on spectrum license costs, and (b) a wide coverage area; a radius of $33 \mathrm{~km}$ that can be increased to $100 \mathrm{~km}$ This is essential in order to reach a large customer base in rural areas. The System architecture of WRAN is a simple point to multipoint system in which a WRAN base station (WRAN BS) serve multiple fixed-location wireless customer premise equipment (CPE) and an associated TV station. Equipped with a directional antenna for communication with BS and an Omni-directional antenna to sense and measure the wireless environmental condition, the CPE is capable of adapting and to timely change the transmission characteristics as necessary in the face of primary user activities.

CRs have the potential to utilize the large amount of unused spectrum in an intelligent way while not interfering with other incumbent devices in frequency bands already licensed for specific uses. CRs are enabled by the rapid and 
significant advancements in radio technologies (e.g., software-defined radios, frequency agility, power control, etc.), and can be characterized by the utilization of disruptive techniques such as wide-band spectrum sensing, real-time spectrum allocation and acquisition, and real-time measurement dissemination [1].

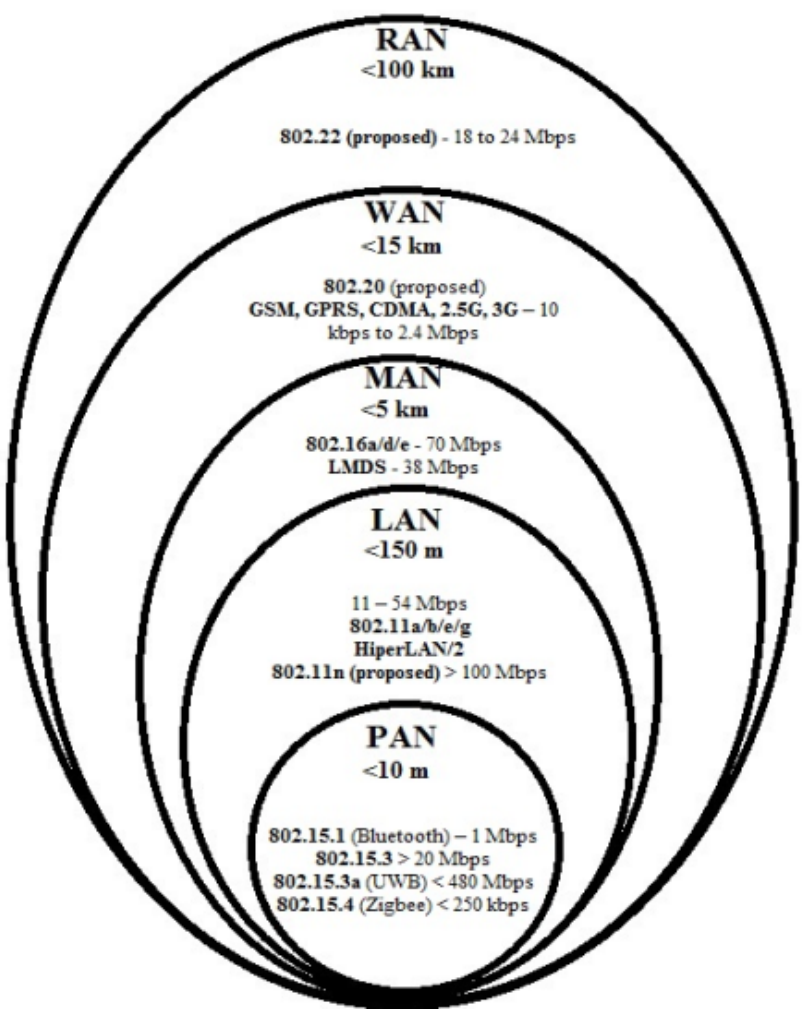

Figure 1. 802.22 wireless RAN classification as compared to other Popular wireless standards

\subsubsection{Main functions of cognitive radio's:}

- Spectrum sensing

- Spectrum management

- Spectrum mobility

- Spectrum sharing

Core Technology-Cognitive radio technology based un-licensed use, primarily designed to operate in the TV Whitespaces from 54-862 MHz, on a non-interfering basis with the primary users (incumbents)

Network Topology-Point-to-Multipoint (PMP)

Sensing antenna-Requires horizontal and vertical polarization sensitivities to sense TV and microphone signals respectively, with Omni-directional pattern.

\subsection{How Rural People Can Access The Broadband Connection with the Help of Cognitive Radio Technology}

Based on the channel availability information, the SM (Spectrum Manager) selects the WRAN's operating channel and backup channel(s) with their corresponding priorities. The algorithm used to Classify and select the operating/backup channels should take into account input from spectrum sensing results from multiple CPEs in order to maximize the channel usage efficiency and guarantee service for the whole cell.

IEEE 802.22: standard for Wireless Regional Area Networks (WRANs) using white spaces in TV spectrum and Rural broadband access which is minimum to use cognitive radio and dynamic spectrum access: must assure that no harmful interference is caused to the incumbent (digital and analog TV broadcasting), and low power licensed devices such as wireless microphones. Wireless Regional Area Network such as IEEE 802.22 system using TV Whitespace can connect rural areas in emerging markets. Television Whitespaces (TVWS) will allow broadband wireless access to regional, rural and remote areas under Line of Sight (LoS) and Non Line of Sight (NLoS) conditions. White Space is a part of the spectrum, available for a radio communication application (service, system) at a given time in a given geographical area on a noninterfering / non-protected basis with regard to primary and other services.

\section{Major Challenges for WRAN}

The IEEE 802.22 standard for wireless regional area network is the first standard for cognitive radio that tries to harness the idle or under-utilized spectrum allocated for TV bands. Two major challenges that are faced by IEEE 802.22 are (i) the issue of self-co-existence and (ii) the hidden incumbent problem [7].

\subsection{Self-co-existence}

In a system like IEEE 802.22 where unlicensed devices are sharing the spectrum under the presence of licensed incumbents, the issue of self-co-existence among multiple IEEE 802.22 operators in an overlapping region is very significant. In areas with high analog/digital TV transmissions and wireless microphone services, unused channels are already commodities of demand. Therefore, when multiple unlicensed operators are operating using a small available band of frequency, there is a chance that the operators will try to act greedy and occupy the available bandwidth. As all the operators will act in the same way, this may result in interference among IEEE 802.22 networks themselves. Thus an efficient channel allocation method needs to be invoked in order to use the channels with least interference. Although the exact methodology for interference Mitigation in IEEE 802.22 networks is yet unknown, we propose an algorithm that increases the spectrum utilization [3].

As shown in Figure 2, several WRAN cells may overlap in their working vicinities [7]. The resultant interference between these overlapped cells leads to one of the major challenges in WRAN systems, namely coexistence problem. This problem may degrade the performance of the system due to the fact that the WRAN coverage range can go up to 
$100 \mathrm{Km}$. As a result, the interference range of this WRAN cell is larger than that in any existing unlicensed technology. Furthermore, a WRAN system opportunistically operates in an unlicensed spectrum, unlike other systems such as cellular systems in which operators use a certain portion of their licensed spectrum. Therefore, coordination between different BSs is needed.

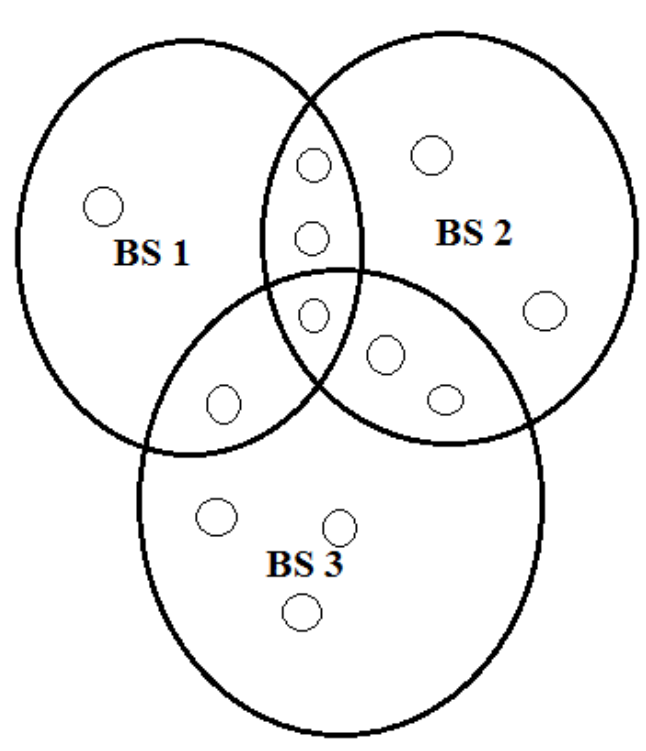

Figure 2. Coexistence problem in IEEE 802.22.

\subsection{Hidden Incumbent Problem}

Let us assume that a BS and a CPE are communicating using a specific frequency channel and an incumbent returns to the same frequency channel near the CPE but outside the BS sensing region (refer Fig. 3 - hidden incumbent region). The CPE can detect the incumbent transmission in-band, but the BS cannot. The BS will continue transmission and might interfere with the incumbent. The CPE cannot report this licensed incumbent as its transmission will cause interference to the incumbent. On the other hand, due to the centralized nature of the IEEE 802.22 network (on-air activities of CPE is controlled by $\mathrm{BS})$, the CPE cannot choose any other channel to connect to the BS as it is not permitted to use any other channel unless BS provides the permission. The problem gets worse as the CPEs do not have any reporting period. Instead what they do have is a channel move time ( 2 seconds) which means that if they sense any incumbent present in the same frequency band they have to move within the stipulated channel move time [4].

In the context of IEEE 802.22 networks, the hidden incumbent problem refers to a situation in which consumer premise equipment (CPE) is within the protection region of an operating incumbent but it fails to report the existence of the incumbent to its base station (BS). The BS transmits in the incumbent's band but is unaware of the fact that the incumbent is using the same band. In such a scenario, CPEs within the incumbent's transmission range may not be able to decode the BS signal because of the strong interference from the incumbent signal. Moreover, the CPEs cannot report the existence of the incumbent as their transmission will cause interference to the incumbent. Therefore, the BS fails to detect the presence of the incumbent.

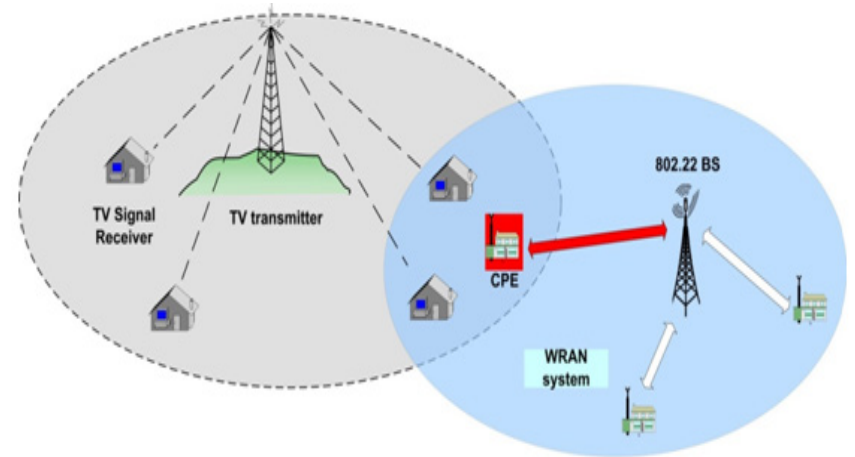

Figure 3. The hidden incumbent problem

\section{Comparison between IEEE 802.16e and IEEE 802.22 Standards [5]}

Table 1. Comparison between IEEE 802.16e and IEEE 802.22 standards

\begin{tabular}{|l|l|l|}
\hline & IEEE 802.16E & IEEE 802.22 \\
\hline Coverage range & $1-5 \mathrm{Km}$ & $33-100 \mathrm{Km}$ \\
\hline Air Interference & $\begin{array}{l}\text { OFDMA, OFDM, } \\
\text { single carrier }\end{array}$ & OFDMA \\
\hline $\begin{array}{l}\text { Multiple Antenna } \\
\text { techniques }\end{array}$ & $\begin{array}{l}\text { Support } \\
\text { Multiplexing, } \\
\text { space time coding } \\
\text { and Beam forming }\end{array}$ & Not supported \\
\hline Coexistence with & Not supported & $\begin{array}{l}\text { Spectrum sensing } \\
\text { management, }\end{array}$ \\
\hline incumbent & $\begin{array}{l}\text { Geo location } \\
\text { management, } \\
\text { incumbent } \\
\text { database query } \\
\text { and channel } \\
\text { management }\end{array}$ \\
\hline $\begin{array}{l}\text { OFDMA channel } \\
\text { profile (MHz) }\end{array}$ & $\begin{array}{l}28,20,17.5,14, \\
10,75,7,3.5, \\
1.25\end{array}$ & $\begin{array}{l}6,7,8 \text { (according to } \\
\text { Regulatory } \\
\text { Domain) }\end{array}$ \\
\hline Self-coexistence & $\begin{array}{l}\text { Master frame } \\
\text { assignment }\end{array}$ & $\begin{array}{l}\text { Dynamic spectrum } \\
\text { assignment }\end{array}$ \\
\hline
\end{tabular}

\section{Conclusion}

This IEEE 802.22 is a standard for Wireless Regional Area Network (WRAN) using white spaces in the TV frequency spectrum. Telco's in the U.S. that serve rural areas and network operators in countries with sparsely 
populated or geographically dispersed users (e.g. Australia, Canada, South America, etc.) will seriously consider early deployment of 802.22 - based WRANs. Fixed line long distance Telco's will consider using WRANs to broaden their networks reach by creating broadband wireless tail circuits to business customers. Fixed Wireless Access (FWA) (and hence WRAN) provides access to one or more (public and private) core networks, rather than forming an end-to-end communication system. 802.22 systems serve fixed location customers, but who might be geographically fixed, re-locatable or nomadic. Cognitive radio networks can utilize the spectrum more efficiently, but they require new regulatory frameworks that enable dynamic spectrum access models. Amongst dynamic spectrum access models, the shared usage model with primary users can be realized with existing cognitive radio technologies. So, in our planning a huge number of subscribers can be connected to broadband access in suburban areas which is not possible by WIMAX. The 802.22 standard is being developed to harness these new opportunities and provide a cost-effective wireless alternative to wired broadband access, especially in rural communities. Hence, the work that is being done in the 802.22 WG will have a huge potential market.

\section{Acknowledgements}

We are thankful to GMR Institute of Technology, Rajam, AP, India and all our faculty members of Information Technology Department for their encouragement and whole hearted cooperation. We would also like to thankful to Ms.Geetha R.B (Asso.Professor \&HOD IT/MCA) for her encouragement. We are lucky to have god like Parents, they provided me very excellent moral support and Friends for their encouragement during this work.

\section{REFERENCES}

[1] Carlos Cordeiro, Kiran Challapali, and Dagnachew Birru,IEEE 802.22: An Introduction to the First Wireless Standard based on Cognitive Radios, JOURNAL OF COMMUNICATIONS, VOL. 1, NO. 1, APRIL 2006.

[2] http://www.ieee802.org/22/, IEEE 802.22 WRAN website.

[3] Raed Al-Zubi, Mohammad Z. Siam, and Marwan Krunz, Coexistence Problem in IEEE 802.22 Wireless Regional Area Networks.

[4] Kaigui Bian and Jung-Min "Jerry" Park, ADDRESSING THE HIDDEN INCUMBENT PROBLEM IN 802.22 NETWORKS, the SDR '09 Technical Conference and Product Exposition.

[5] Santa Rahman, Nahid Hossain, Nizam Sayeed, M.L. Palash, Comparative Study between Wireless Regional Area Network (IEEE Standard 802.22) and WiMAX and Coverage Planning of a Wireless Regional Area Network Using Cognitive Radio Technology, International Journal of Recent
Technology and Engineering (IJRTE) ISSN: 2277-3878, Volume-1, Issue-6, January 2013.

[6] http://www.wikipedia.org.

[7] Timothy X Brown, Douglas C. Sicker,Can Cognitive Radio Support Broadband Wireless Access?, 1-4244-0663-3/07/\$20.00 C2007 IEEE.

[8] Mardeni R. \& Chimheno R. L., Wireless Regional Access Networks: A Wise Choice for InternetConnectivity to Rural Areas of Zimbabwe, Modern Applied Science; Vol. 7, No. 7; 2013,ISSN 1913-1844 E-ISSN 1913-1852,Published by Canadian Center of Science and Education.

[9] Dave Cavalcanti and Monisha Ghosh, Cognitive Radio Networks: Enabling New Wireless Broadband Opportunities,IEEE, E-ISBN :978-1-4244-2302-6.

[10] J. Mitola et al., "Cognitive Radios: Making Software Radios more Personal," IEEE Personal Communications, vol. 6, no. 4, Aug. 1999.

[11] S. Haykin, "Cognitive Radio: Brain-Empowered Wireless Communications," in IEEE JSAC, vol. 23, no. 2, Feb. 2005.

[12] Shamik Sengupta, Swastik Brahma and Mainak Chatterjee, Sai Shankar N, Enhancements to cognitive radio based IEEE 802.22 air-interface, the direction of IEEE Communications Society. 